the absorption bands at $800-900 \mathrm{~cm}^{-1}$ and at 688 $\mathrm{cm}^{-1}$. This supports the assumption that only the arrangement of the molecules in the crystal is different in both modifications.

The correlation of NQR frequencies with the substituent parameters $*$ is fairly good as demonstrated by Fig. I. The deviations from the straight line $v_{\mathrm{obs}}$ $=v_{\text {calc }}$ are within the limits of $\pm 500 \mathrm{kHz}$. This conforms to the usually assumed value of the crystal field effect. The $\varkappa$-values of the diazonium group decrease in the order ortho>meta>para. The magnitude of the $\varkappa$ 's shows the very strong electron attraction of the diazonium group. From the chemical knowledge (e. g. the activation effect of the $\mathrm{N}_{2}{ }^{\oplus}$. group in ortho- and para-positon toward the aromatic nucleophilic substitution ${ }^{11}$ ) one would expect the order $o>p>m$ because of the strong " $-\mathrm{R}$ ".

11 J. F. Brunnet u. R. E. Zahler, Chem. Reviews 49, 277, 284, 308 [1951]. effect of the electron attracting diazonium group. The $\sigma$-values, derived from dissociation constants of the carboxybenzenediazonium acids by LEwIS and JoHNSON ${ }^{12}$, accord with this chemical expectation. The agreement of the $o$ - and $m-\varkappa$-values with the corresponding $\sigma$-values is unexpectedly good, but the values for the para-positon are significantly different. This can be explained by the crucial influence of the inductive effect, causing the order $o>m>p$, and the insignificance of the mesomeric effect to the NQR-experiment. This reflects the fact that the ground state of the molecule determines NQR measurements, while in chemical experiments excited states of the molecule may often be decisive.

We are grateful to Mr. W. WEYRICH for his help during the least-squares calculations and to the Deutsche Forschungsgemeinschaft for financial support.

12 E. S. Lewis u. M. D. Johnson, J. Amer. chem. Soc. 81, 2070 [1959].

\title{
Chemische Untersuchungen an Chondroitinsulfat- und Heparansulfat-Peptiden aus menschlichen Aorten IV
}

\author{
A. Klemer, D. Mempel und K. Römer \\ Organisch-Chemisches Institut der Universität Münster (Westf.) \\ (Z. Naturforschg. 24 b, 1273-1279 [1969]; eingegangen am 27. Februar 1969)
}

\begin{abstract}
Unabhängig vom Lebensalter und Sklerosegrad enthalten menschliche Aorten gleichartig aufgebaute Chondroitinsulfat- und Heparansulfat-Peptide, wobei das Verhältnis der ChondroitinsulfatPeptide A und C etwa konstant bleibt.

Der Gehalt menschlicher Aorten an Chondroitinsulfat-Peptiden ist in Abhängigkeit vom Lebensalter und Sklerosegrad verschieden. Den höchsten Gehalt hat die Aorta bei beginnender Sklerose. Einen gleichartigen Gang in der quantitativen Verteilung findet man beim Heparansulfat-Peptid.
\end{abstract}

Die Arteriosklerose wird durch eine Erhöhung des Sulfatstoffwechsels des Gewebes eingeleitet ${ }^{1}$. Erst sekundär kommt es zu den bekannten Ablagerungen von Cholesterin und Ca-Phosphat an der Intima. Demgegenüber nimmt im gesunden Gewebe der Sulfatstoffwechsel mit steigendem Lebensalter $a b$, ein Befund, der dem allgemein verlangsamten Stoffwechsel im Alter entspricht.

Wie wir früher berichteten ${ }^{2,3}$, enthalten menschliche normale und sklerotische Aorten Chondroitin-

Abkürzungen: $\mathrm{CS}=$ Chondroitinsulfat, CS-A $=$ Chondroitin-4-sulfat, CS-C = Condroitin-6-sulfat, CS-B = Dermatansulfat, HMS = Heparansulfat, HS = Hyaluronsäure.

1 W. H. Hauss u. G. Junge-HüLsing, Dtsch. med. Wschr. 86, 763 [1961]; W. H. Hauss, Verh. dtsch. Ges. innere Med., 69. Kongreß 1963; zusammenfassende Darstellung.
sulfat-Protein-Komplexe (CS-Proteine), die sowohl in der Zusammensetzung ihrer Protein- und Polysaccharid-Komponenten als auch im Mol.-Gew. identisch sind. Sklerotische Aorten enthalten jedoch im Mittel ca. 30\% mehr dieses C.S-Proteins als die normalen. Der Primärschritt der Arteriosklerose schließt demnach eine vermehrte Bildung normal aufgebauten CS-Proteins ein.

Im folgenden berichten wir über den quantitativen Gehalt menschlicher Aorten definierten Alters

2 A. Klemer u. K. Homberg, Liebigs Ann. Chem. 678, 160 [1964].

3 A. Klemer u. D. Mempel, Z. Naturforschg. 20 b, 553 [1965]; A. Klemer, K. Homberg u. D. Mempel, 19 b, 961 [1964]. 
und Sklerosegrades an Chondroitinsulfat- und Heparansulfat-Peptiden sowie die Zusammensetzung dieser Verbindungen.

\begin{tabular}{|c|c|c|c|c|}
\hline $\begin{array}{c}\text { Be- } \\
\text { zeich- } \\
\text { nung * }\end{array}$ & $\begin{array}{c}\text { Sklerose- } \\
\text { grad }\end{array}$ & $\begin{array}{l}\text { An- } \\
\text { zahl }\end{array}$ & $\begin{array}{l}\text { enten } \\
\text { Alter }\end{array}$ & $\begin{array}{c}\text { aceton- } \\
\text { trockenes } \\
\text { Aorten- } \\
\text { material } \\
{[\mathrm{g}]}\end{array}$ \\
\hline N 1 & $\begin{array}{c}\text { keine } \\
\text { Sklerose }\end{array}$ & 6 & $20-44$ & 12,1 \\
\hline $\mathrm{N}_{2}$ & & 6 & $17-35$ & 10,7 \\
\hline N 3 & $\begin{array}{l}\text { keine } \\
\text { Sklerose }\end{array}$ & 6 & $15-27$ & 16,3 \\
\hline g A 1 & geringe & 4 & $40-73$ & 13,7 \\
\hline $\mathrm{g}$ A 2 & $\begin{array}{l}\text { geringe } \\
\text { Sklerose }\end{array}$ & 5 & $50-60$ & 23,5 \\
\hline g A 3 & $\begin{array}{l}\text { geringe } \\
\text { Sklerose }\end{array}$ & 3 & $50-61$ & 12,9 \\
\hline $\mathrm{m} \mathrm{A} 1$ & $\begin{array}{l}\text { mäßige } \\
\text { Sklerose }\end{array}$ & 5 & $54-76$ & 23,6 \\
\hline $\mathrm{m} \mathrm{A} 2$ & $\begin{array}{l}\text { mäßige } \\
\text { Sklerose }\end{array}$ & 5 & $52-64$ & 22,0 \\
\hline s A 1 & $\begin{array}{l}\text { schwere } \\
\text { Sklerose }\end{array}$ & 4 & $56-84$ & 17,2 \\
\hline s A 2 & $\begin{array}{l}\text { schwere } \\
\text { Sklerose }\end{array}$ & 3 & $59-78$ & 13,2 \\
\hline
\end{tabular}

Tab. 1. Übersicht über die Herkunft des untersuchten Aortenmaterials. * Fortlaufende arabische Zahlen bezeichnen die einzelnen Versuchsansätze.

Das Aortenmaterial wurde uns von der Med. Klinik, Münster, bereitgestellt. Es war spätestens 24 Stdn. nach dem Tode der Patienten entnommen und sofort in Aceton gelegt worden. Jede Gruppe bestand aus dem Aortenmaterial von 5-7 Patienten bestimmten Alters und Sklerosegrades (s. Tab. 1). Auf Grund der ermittelten Identität der CS-Proteine verschiedener Herkunft (vgl. l. c. ${ }^{2,3}$ ) haben wir bei den im folgenden beschriebenen Untersuchung die Protein-Anteile durch Papain abgebaut. Dies erleichtert die weitere Aufarbeitung wesentlich. Beim Abbau gehen die Aorten zu rd. 90\% len aus chloroformlöslichen Substanzen, in denen sich Cholesterin nachweisen läßt. Außerdem enthalten die Rückstände - wie zu erwarten - Ca-Phosphat. Aus den löslichen Anteilen der Aorten erhalten wir nach dem Ausdialysieren und Fällen unter bestimmten Bedingungen ein rohes MucopolysaccharidPeptid-Gemisch, welches auch die in den Aorten vorkommenden Glykopeptide enthält. Durch Chromato-

${ }^{4}$ S. Schiller, G. A. Slower u. A. Dorfman, J. biol. Chemistry 236, 983 [1961]. graphie an Dowex I-X $2{ }^{4}$ zerlegen wir das Gemisch in seine Komponenten, wobei wir zunächst mit Wasser die neutralen Glykopeptide ${ }^{5}$ und sodann mit $\mathrm{NaCl}$-Lösungen steigender Konzentration die verschiedenen Mucopolysaccharid-Peptide eluieren. Abb. 1 zeigt einen Ausschnitt aus einer Trennung der Gruppe ,geringe Sklerose (gA2) “. Mit l-n. NaCl wird Heparansulfat-Peptid (HMS-Peptid) und mit 1,5-n. bzw. 2-n. $\mathrm{NaCl}$ werden die CS-Peptide A, B und $\mathrm{C}$ eluiert. Die mit 1,25-n $\mathrm{NaCl}$ eluierte kleine Zwischenfraktion ist noch unbekannt. Sie enthält sowohl Glucosamin als auch Galactosamin. Mit der Reindarstellung und Baustein-Analyse befassen wir uns z. Z. noch.

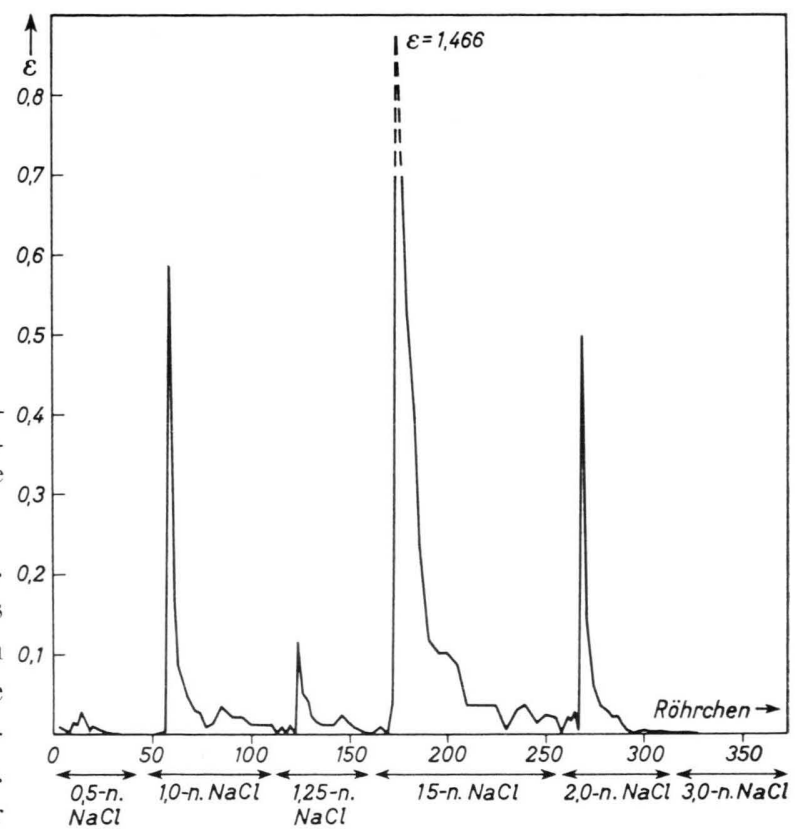

Abb. 1. Ausschnitt aus der Auftrennung der Sulfomucopolysaccharid-Peptide aus der Aortengruppe: geringe Sklerose an Dowex 1-X2 (Einzelheiten s. Versuchsteil).

Alle Fraktionen werden durch den AminosäureAnalysator ausgewertet. Das Spektrum liefert nicht nur quantitativ die noch vorhandenen Aminosäuren, sondern auch ziemlich genau den Gehalt an Aminozuckern.

Auf diese Weise läßt sich aus dem Glucosaminwert auf die HMS-Peptid-Menge und aus dem Galactosamin- und damit im Zusammenhang stehenden Talosaminwert auf die Ausbeute an CS-Peptid l. c. ${ }^{3}$ schließen. Die Uronsäurebestimmung nach

5 A. Klemer u. C. Burbaum, Z. Naturforschg. 20 b, 712 [1965] ; A. Klemer u. C. Nager, 22 b, 456 [1967]. 
der Carbazolmethode liefert in allen Fällen Werte, die den gefundenen Aminozuckermengen etwa entsprechen.

Unabhängig davon haben wir die Einheitlichkeit der Fraktionen auf papierelektrophoretischem Wege mit einem von uns entwickelten System ${ }^{6}$ überprüft.

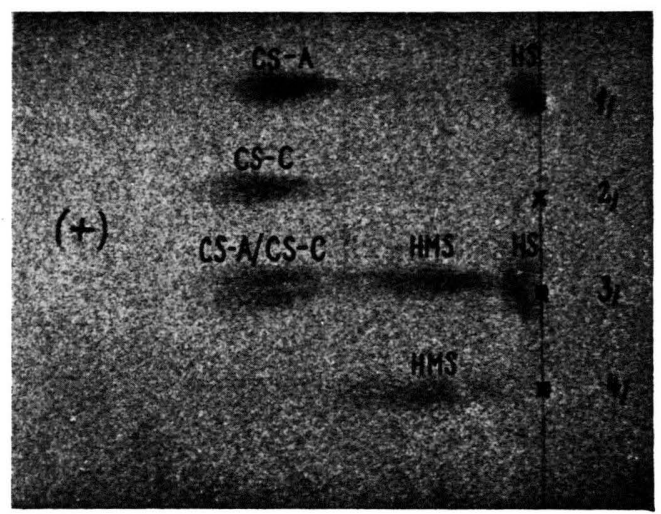

Abb. 2. Schleicher \& Schüll Nr. 2043/45 ac, Essigsäure-Ameisensäurepuffer $(\mathrm{pH} 1,9) 1 \mathrm{~mA} / \mathrm{cm}, 1$ Stde. Laufzeit, Toluidinblau 1. $10 \gamma$ CS-A-Peptid $+30 \gamma$ HS, $2.10 \gamma$ CS-C-Peptid, $3.10 \gamma$ CS-A- $+10 \gamma$ CS-C- $+30 \gamma$ HMS-Peptid $+30 \gamma$ HS, 4. $30 \gamma$ HMS-Peptid.

Abb. 2 zeigt eine Trennung, die auf hochacetyliertem Papier mit einem Essigsäure-Ameisensäurepuffer erzielt wurde. CS- und HMS-Peptid kann man deutlich unterscheiden. Auch die Hyaluronsäure, hier nahe am Startpunkt, hebt sich deutlich ab. Dies ist besonders wichtig, weil eine Verunreinigung des HMS-Peptids durch Hyaluronsäure, die beim Trennungsgang an Dowex 1-X 2 unmittelbar vor dem HMS-Peptid auftritt, häufig beobachtet wird. Durch das Aminozuckerspektrum kann man diese Verunreinigung nicht erkennen, da beide Mucopolysaccharide sich ja von Glucosamin ableiten.

Charakterisierung der CS- und HMS-Peptide der Aortengruppen: keine Sklerose (N), geringe Sklerose (gA), mäßige Sklerose ( $m A$ ) und starke Sklerose ( $s A)$

1. Aminosäuregehalte (vgl. Tab. 2)

\section{a) CS-Peptide}

Alle CS-Peptide haben nach dem Papain-Abbau noch einen Aminosäure-Gehalt von rd. 1,0 Prozent. Dieser verteilt sich auf 7 Aminosäuren in merklichen Konzentrationen. Die Zahlen in Tab. 2 geben die Molverhältnisse wieder, wobei auf Glutaminsäure $=2$ bezogen ist. Wie man sieht, werden in allen Proben etwa die gleichen Verhältnisse gefunden, ein Befund, der den gleichartigen Aufbau der aus normalen und sklerotischen Aorten isolierten CS-Peptide bestätigt (vgl. l. c. ${ }^{3}$ ) .

Auffällig ist, daß Glykokoll und Alanin gleichmäßig in größeren Mengen vertreten sind. Da bis-

\begin{tabular}{|c|c|c|c|c|c|c|c|c|}
\hline \multirow{2}{*}{$\begin{array}{l}\text { Amino- } \\
\text { säuren }\end{array}$} & \multicolumn{4}{|c|}{$\begin{array}{c}\text { CS-Peptide } \\
\text { Amina }\end{array}$} & \multicolumn{4}{|c|}{ HMS-Peptide } \\
\hline & $\mathrm{N}$ & gA & $\mathrm{mA}$ & $\mathrm{sA}$ & $\mathrm{N}$ & gA & $\mathrm{mA}$ & sA \\
\hline Lys & - & - & - & - & - & - & - & - \\
\hline $\mathrm{His}$ & - & - & - & - & - & - & - & - \\
\hline Arg & - & - & - & - & - & - & - & - \\
\hline Asp & 2 & 2 & 2 & 2 & $2(3)$ & $2(3)$ & $2(3)$ & 3 \\
\hline Thr & 1 & 1 & 1 & 1 & $1(0)$ & $1(0)$ & $1(0)$ & $1(0)$ \\
\hline Ser & 4 & $4(5)$ & $3(4)$ & 4 & 4 & 4 & 4 & 4 \\
\hline Glu & 2 & 2 & 2 & $2(3)$ & 2 & 2 & 2 & 2 \\
\hline Pro & - & $1(0)$ & - & 1 & - & - & - & - \\
\hline Gly & 3 & $3(4)$ & $3(4)$ & $3(4)$ & 4 & $4(5)$ & 4 & 4 \\
\hline Ala & 2 & 2 & 2 & 2 & 2 & $2(1)$ & $2(1)$ & $2(1)$ \\
\hline Cys & - & - & - & - & - & - & - & - \\
\hline Val & - & $1(0)$ & $1(0)$ & - & - & - & - & - \\
\hline Met & - & - & - & - & - & - & - & - \\
\hline Ile & $1(0)$ & $1(0)$ & $1(0)$ & $1(0)$ & - & - & - & - \\
\hline Leu & 1 & 1 & 1 & 1 & $1(0)$ & $1(0)$ & $\mathbf{l}(0)$ & - \\
\hline Tyr & - & - & - & - & - & - & - & - \\
\hline Phe & - & - & - & - & - & - & - & - \\
\hline
\end{tabular}

Tab. 2. Aminosäuregehalte der CS- und HMS-Peptide (Molverhältnisse). Die in Klammern angegebenen Zahlen geben gelegentlich gefundene Werte an.

6 Diplom-Arbeit, W. Fuhrmann, Münster-Westf. 1967.

7 G. Blix, Ann. N.Y. Acad. Sci. 106, 164 [1963].
8 E. R. B. Graham, W. N. Murphy u. A. Gottschalk, Biochim. biophysica Acta [Amsterdam] 74, 222 [1963]. 
her in Kohlenhydrat-Eiweißverbindungen ein solcher Verknüpfungstyp nicht gefunden wurde, ist daran zu denken, daß diese beiden Aminosäuren regelmäßig in näherer Umgebung der mit Kohlenhydrat verknüpften Hydroxyaminosäuren und Aminodicarbonsäuren anzutreffen sind. Für Glykoproteide der Submaxillaris-Gruppe ist die Nachbarschaft von Glykokoll und Alanin zu den Hydroxyaminosäuren bewiesen worden 7,8 .

\section{b) HMS-Peptide}

Wie die Tab. 2 zeigt, besitzen auch die verschiedenen HMS-Peptide unabhängig vom Sklerosegrad und Alter der Patienten den gleichen Aminosäuregehalt, sowohl qualitativ als auch annähernd quantitativ. Auffällig ist die große Ähnlichkeit des Aminosäure-Musters im Vergleich zu dem der CSPeptide. Der Befund spricht dafür, daß dieses Mucopolysaccharid an ein sehr ähnlich strukturiertes Protein im Bereich der Verknüpfungspunkte zum Polysaccharid gebunden ist.

\section{Die Polysaccharid-Komponenten der CS-Peptide}

Nach der in dieser Arbeit beschriebenen Aufarbeitung erhalten wir Gemische aus CS-A-, CS-B- * und CS-C-Peptid. Dies zeigt auch die IR-spektroskopische Untersuchung der Proben. Es werden die charakteristischen Sulfat-Banden bei $850 \mathrm{~cm}^{-1}$ (CS-A, CS-B) und $820 \mathrm{~cm}^{-1}$ (CS-C) gefunden ${ }^{9}$.

Zwecks Ermittlung der quantitativen Verteilung von CS-C- und CS-A bzw. CS-B-Peptiden in Aorten in Abhängigkeit von Sklerosegrad und Lebensalter haben wir den CS-C-Gehalt unserer verschiedenen CS-Peptid-Gemische nach der von MATHEws entwickelten Methode ${ }^{10}$ durch Hyaluronidase-Abbau kombiniert mit Hexosamin-Bestimmungen vor und nach dem Abbau bestimmt. Wie Tab. 3 zeigt, bleibt das Verhältnis von CS-C-: CS-A,B-Peptid unabhängig von Sklerosegrad und Lebensalter etwa konstant. Dieser Befund steht im Gegensatz zu den Ergebnissen von Kaplan und Meyer ${ }^{11}$, die in menschlichen Aorten eine Abnahme von CS-C mit steigendem Lebensalter feststellten. Demgegenüber zeigen

* Über die Isolierung und Zusammensetzung von CS-B-Peptid werden wir später berichten.

9 M. B. Mathews, Nature [London] 181, 421 [1958].

10 M. B. Mathews u. M. Inouye, Biochim. biophysica Acta [Amsterdam] 53, 509 [1961].

\begin{tabular}{|c|c|c|c|c|}
\hline $\begin{array}{l}\text { Versuchs- } \\
\text { bezeichnung } \\
\text { siehe Tab. } 1\end{array}$ & $\begin{array}{c}\text { normale } \\
\text { Aorten } \\
(\mathrm{N} \text { l) } \\
{[\%]}\end{array}$ & $\begin{array}{c}\text { geringe } \\
\text { Sklerose } \\
(\mathrm{g} \mathrm{A} \mathrm{1}) \\
{[\%]}\end{array}$ & $\begin{array}{c}\text { mäßige } \\
\text { Sklerose } \\
(\mathrm{m} \mathrm{A} \mathrm{l}) \\
{[\%]}\end{array}$ & $\begin{array}{c}\text { starke } \\
\text { Sklerose } \\
\text { (s A 1) } \\
{[\%]}\end{array}$ \\
\hline $\begin{array}{l}\text { Rückstand nach } \\
\text { Papain-Abbau } \\
\Sigma \text { ABC } \\
\text { Gesamt-CS- } \\
\text { Peptid* } \\
\text { (CS-C-,-A- u. -B- } \\
\text { Peptid) } \\
\text { davon CS-C- } \\
\text { Peptid ** }\end{array}$ & 0,5 & 12,7 & 12,2 & $\begin{array}{r}0,7 \\
60,5\end{array}$ \\
\hline
\end{tabular}

Tab. 3. Gehalt menschlicher Aorten (gestaffelt nach Sklerosegrad und Lebensalter) an Gesamt-CS-Peptid und CS-C-Peptid. * Bezogen auf acetonbehandeltes, getrocknetes Aortenmaterial abzüglich Rückstand $\Sigma$ ABC (vgl. Tab. 4). ** Fehlerbreite $10 \%$, l. c. ${ }^{10}$.

Untersuchungen am menschlichen Zwischenwirbel außer einer Abnahme an Gesamtchondroitinsulfat eine Verschiebung der Relation CS-A/CS-C zugunsten von CS-C mit steigendem Lebensalter ${ }^{12}$.

Quantitative Verteilung der CS- und HMS-Peptide in den Aortengruppen: keine Sklerose (N), geringe Sklerose ( $\mathrm{g} A)$, mäßige Sklerose $(m A)$ und starke Sklerose (sA) (vgl. Tab. 4)

Angaben über die quantitative Verteilung der Sulfomucopolysaccharide sind nur sinnvoll, wenn das verwendete Aortenmaterial in definierter Form vorliegt. Diese Voraussetzung ist nicht gegeben, wenn auf das acetonbehandelte und getrocknete Sektionsgut bezogen wird. Es werden nämlich, wie wir feststellten, wechselnde Mengen Cholesterin und Lipoide, abhängig vom Sklerosegrad des Sektionsgutes durch die mehrfache Acetonbehandlung extrahiert. Demgemäß erhält man nur Relativwerte, wenn auf dieses Material bezogen wird (vgl. Teil 2 der Tab.4 CS- und HMS-Peptid-Werte unter a). Definiertere Verhältnisse liegen vor, wenn das Sektionsgut abzüglich seines Gesamtgehaltes an Cholesterin, Lipoiden und anorganischen Bestandteilen als Bezugssystem zu Grunde gelegt wird (vgl. Angaben unter b).

Hierzu haben wir die nach dem Papain-Abbau des acetonbehandelten Sektionsgutes verbleibenden Rückstände, die im wesentlichen aus Cholesterin

11 D. Kaplan u. K. Meyer, Proc. Soc. exp. Biol. Med. 105, 78 [1960].

12 E. Buddecke u. M. Sziegoleit, Hoppe Seyler's Z. physiol. Chem. 337, 66 [1964]. 


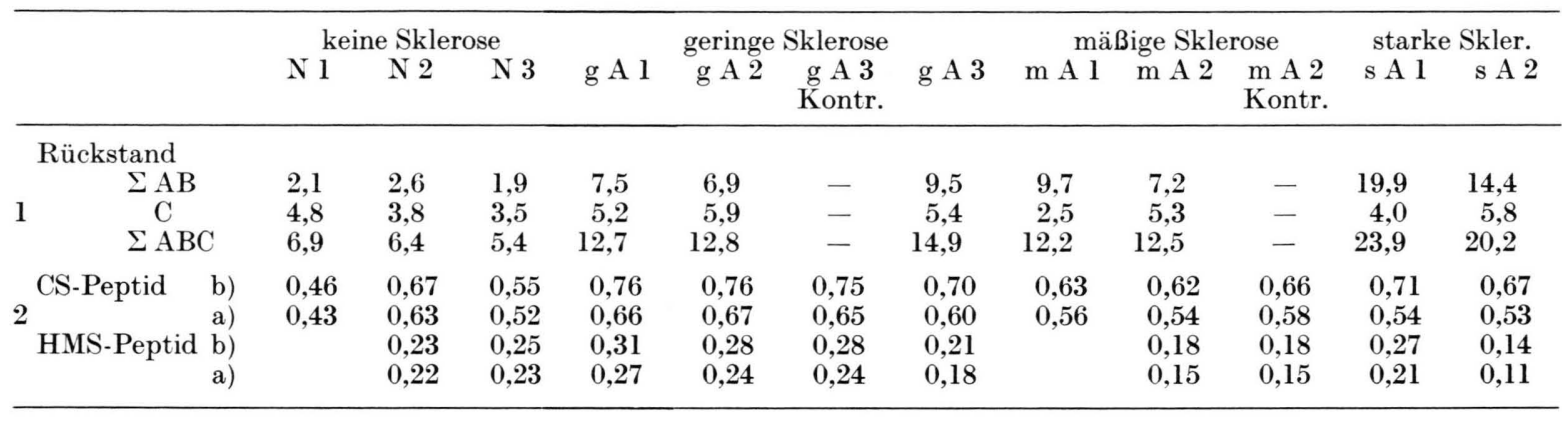

Tab. 4. Gehalt menschlicher Aorten (gestaffelt nach Sklerosegrad) an CS- und HMS-Peptiden (Angabe in \%). Zu Teil 1: Rückstand $\mathrm{AB}$ und $\mathrm{C}$ bez. auf acetonbehandeltes, getrocknetes Aortenmaterial. Zu Teil 2: a) = bez. auf acetonbehandeltes, getrocknetes Aortenmaterial, b) $=$ bez. auf acetonbehandeltes, getrocknetes Aortenmaterial $-\mathrm{Rückstand} \Sigma \mathrm{ABC}$, $\mathrm{AB}=$ Mineralien, Cholesterin, $\mathrm{C}=$ Lipopolysaccharide (alkohollöslich).

und Ca-Phosphat bestehen, in Abzug gebracht (vgl. Teil 1 der Tab. 4 AB). In der Gruppe der Patienten mit starker Sklerose beträgt dieser Anteil im Mittel $16,5 \%$ gegenüber nur $2,2 \%$ in der Gruppe der jungen, sklerosefreien Menschen.

Ferner haben wir einen alkohollöslichen Anteil (Fraktion C) berücksichtigt, der im Rahmen der Aufarbeitung anfällt. Überwiegend scheint diese Fraktion aus Lipoiden, uronsäurefreien Polysacchariden und Eiweißstoffen zu bestehen. Dieser Anteil beträgt im Mittel in allen Aortengruppen 4-5 Prozent. In Abb. 3 sind die Gehalte der verschiede-

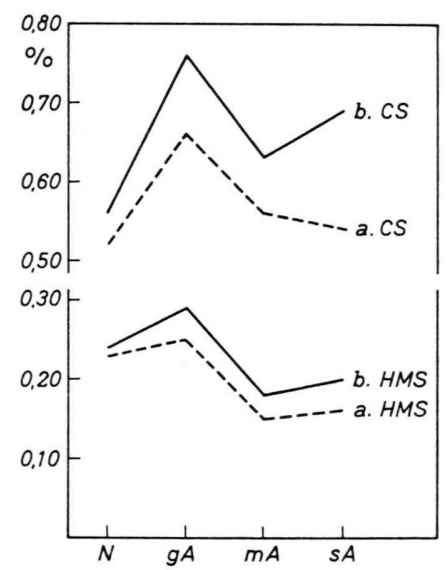

Abb. 3. Quantitative Verteilung von CS- und HMS-Peptiden in Aorten in Abhängigkeit vom Sklerosegrad und Lebensalter. a) \% bez. auf acetonbehandeltes, getrocknetes Aortenmaterial; b) \% bez. auf acetonbehandeltes, getrocknetes Aortenmaterial - Rückstand $\Sigma \mathrm{AB}+$ (vgl. Tab. 4) .

nen Aortengruppen an CS- und HMS-Peptiden nach diesen Gesichtspunkten graphisch zusammengestellt.

\section{a) CS-Peptide}

Wie man sieht, steigt bei geringer Sklerose der CS-Peptid-Gehalt auf seinen höchsten Wert an. Gegenüber dem Normalgehalt nimmt er um mehr als $1 / 3$ zu. Dieser Wert steht mit dem besonders erhöhten Sulfatstoffwechsel, wie er bei beginnender Sklerose - mit fehlenden oder geringfügigen histologischen Veränderungen - beobachtet wird, in guter Übereinstimmung. Überraschend geht jedoch bei mäßiger Sklerose der CS-Peptid-Gehalt erheblich zurück, um bei starker Sklerose erneut anzusteigen, wenn auf das Aortenmaterial ohne Sekundärablagerungen bezogen wird. Berücksichtigt man diesen Rückstand nicht, so ist ein weiterer leichter Abfall zu verzeichnen. Die CS-Peptid-Gehalte in der Aortengruppe mit mäßiger Sklerose $(\mathrm{mA})$ sind wesentlich niedriger als nach der Höhe der Einbaurate an ${ }^{35} \mathrm{~S}$ (Hauss und Mitarb.) erwartet wurde. Da andererseits aber durch Kontrollen (vgl. Tab. 4) die Zuverlässigkeit unserer Trennungen sichergestellt ist, bedarf es zur Klärung dieser Diskrepanz weiterer Untersuchungen. Präparative Trennungen zur quantitativen Isolierung der Sulfomucopolysaccharide aus Aorten mit mäßiger Sklerose, jedoch geordnet nach der Höhe des Sulfatstoffwechsels sind in Vorbereitung.

\section{b) HMS-Peptide}

Die HMS-Peptid-Gehalte menschlicher Aorten sind generell nur etwa $1 / 2$ bis $1 / 3$ so hoch wie die der CS-Peptide. Obwohl damit verbunden die Fehlergrenze größer ist, kann man doch einen grundsätzlich gleichartigen Gang in der Verteilung in den verschiedenen Aortengruppen abschätzen. Daraus ergibt sich, daß dieses Sulfomucopolysaccharid von den Veränderungen im Stoffwechsel in gleicher Weise betroffen ist. 


\begin{tabular}{|c|c|c|c|c|c|c|c|c|}
\hline \multirow[t]{2}{*}{$\begin{array}{l}\text { Fraktion } \\
{[-n . \mathrm{NaCl}]}\end{array}$} & \multicolumn{2}{|c|}{$\begin{array}{c}\text { keine Sklerose } \\
(\mathrm{N} 3) \\
\mathrm{E}^{\prime}: 260\end{array}$} & \multicolumn{2}{|c|}{$\begin{array}{c}\text { geringe Sklerose } \\
\text { (g A 2) } \\
\mathrm{E}^{\prime}: 245\end{array}$} & \multicolumn{2}{|c|}{$\begin{array}{c}\text { mäßige Sklerose } \\
(\mathrm{m} \text { A } 2) \\
\mathrm{E}^{\prime}: 248\end{array}$} & \multicolumn{2}{|c|}{$\begin{array}{c}\text { starke Sklerose } \\
\text { (s A 1) } \\
\mathrm{E}^{\prime}: 218\end{array}$} \\
\hline & CS- & HMS- & $\mathrm{CS}$ - & HMS- & CS- & HMS- & CS- & HMS-Peptid \\
\hline 1,0 & 1,5 & 27,0 & $\begin{array}{c}\text { unter } \\
1,0\end{array}$ & 21,5 & Spur & 15,4 & $\begin{array}{c}\text { unter } \\
1,0\end{array}$ & 11,5 \\
\hline $1,25 *$ & 2,5 & & 2,5 & & 2,0 & & 3,0 & \\
\hline 1,5 & 55,0 & 1,0 & 42,0 & & 44,5 & & 50,0 & 1,0 \\
\hline 2,0 & 16,0 & & 19,5 & & 6,5 & & 5,5 & \\
\hline
\end{tabular}

Tab. 5. Ubersicht über je eine Auftrennung der Aortengruppen N, gA, mA und sA (Angabe in mg). E' $=$ mg eingesetztes Rohmucopolysaccharid. * Zusätzlich findet sich in jeder $1,25-n$. NaCl-Fraktion in sehr geringer Menge $(0,8-1,7 \mathrm{mg})$ einc noch unbekannte Substanz.

\section{Beschreibung der Versuche}

$$
\text { A usgangsmaterial }
$$

Das Aortenmaterial wurde von der Med. Klinik der Universität Münster, Prof. Dr. Hauss, bereitgestellt. Die Aorten wurden spätestens 24 Stdn. nach dem Tode des Patienten dem Sektionsgut entnommen und nach dem skerotischen Befund und Alter geordnet. Fett und Bindegewebe wurden abgetrennt, die Aorten zerkleinert und bei Zimmertemperatur in Aceton gelegt, das mehrmals erneuert wurde. Für die Aufarbeitung l. c. ${ }^{2,3}$ wurde das acetontrockene Material eingesetzt.

$$
\text { Analytische Methoden }
$$

Vor den Bestimmungen wurden die zu untersuchenden Substanzen ca. $15 \mathrm{Stdn}$. im Hochvak. bei $40^{\circ}$ bis zur Gewichtskonstanz getrocknet.

Uronsäurebestimmung: Nach Dische ${ }^{13}$ durch photometrische Auswertung.

Hexosaminbestimmung: Nach Schultze, SchmidtBERger und HAUPT ${ }^{14}$. Die Proben wurden zuvor 4. Stdn. bei $105^{\circ}$ in 6-n. $\mathrm{HCl}$ hydrolysiert.

Aminosäure- und Hexosaminbestimmung im Aminosäureanalysator - Beckman-Spinco, Modell 120: Die Proben wurden 3 (speziell für Hexosaminbestimmung) oder $8 \mathrm{Stdn}$. in geschlossenen Kölbchen bei $100^{\circ}$ in 6- $n$. $\mathrm{HCl}$ hydrolysiert.

Bestimmung von CS-C (vgl. 1. c. ${ }^{10}$ ): Der Abbau wurde mit Hyaluronidase aus Schaftestes (500 IE mg) vorgenommen. Hexosamin- bzw. $N$-Acetyl-hexosamin wurden wie in 1. c. ${ }^{10}$ angegeben, bestimmt.

Isolierung von CS und HMS-Peptiden: $10-25 \mathrm{~g}$ mit Aceton entfettetes und getrocknetes Aortenmaterial werden in $300-400 \mathrm{ml}$ Wasser durch Rühren mit dem Ultra-Turrax (Typ T45 6) zerkleinert. Hierzu gibt man in Anlehnung an die Vorschrift von BudDECKE und Mitarbb. ${ }^{15}$ soviel Puffersubstanz, daß die Lösung eine Konzentration von 0,1-m. Natriumacetat, 0,005-m. Cystein-HCl und 0,005 Komplexon III aufweist; ferner

13 Z. Dische, J. biol. Chemistry 167, 189 [1947].

14 H. E. Schultze, R. Schmidtberger u. H. Haupt, Biochem. Z. 329, 497 [1958].

15 E. Buddecke, W. KröZ u. E. Lanka, Hoppe-Seyler's Z. physiol. Chem. 331, 196 [1963]. werden pro g Aortenmaterial $2 \mathrm{mg}$ Papain * zugesetzt. Die Inkubationszeit beträgt $24 \mathrm{Stdn}$. bei $60^{\circ}$, wobei mit wenig Toluol überschichtet wird. Hiernach wird zentrifugiert und der Niederschlag erneut, wie oben angegeben, mit Papain abgebaut. Der unlösliche Rückstand wird abzentrifugiert, mit Wasser gewaschen und gefriergetrocknet (Fraktion A)**. Die vereinigten Überstände werden dialysiert, eingeengt und nochmals bei $15000 \mathrm{U} / \mathrm{min}$ mit einer Pirouette zentrifugiert (Fraktion B) ${ }^{* *}$. Der Überstand wird mit der 3-fachen Menge Äthanol und $1 \mathrm{~g}$ Kaliumacetat pro $100 \mathrm{ml}$ Lösung versetzt. Nach mehrstündigem Stehen bei $4^{\circ}$ wird erneut

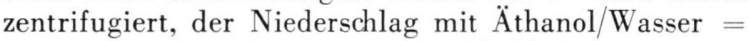
$3: 1$ gewaschen, in einer $1,5-n$. $\mathrm{MgCl}_{2}$-Lösung aufgenommen, zentrifugiert und der Überstand erneut mit Äthanol/Kaliumacetat gefällt. Nach Stehen in der Kälte wird der abzentrifugierte Niederschlag in Wasser aufgenommen, an einer Säule $(40 \times 1,5 \mathrm{~cm}) \mathrm{mit}$ Amberlite IR $120\left[\mathrm{H}^{\oplus}\right.$-Form] gereinigt und in die freie Säure übergeführt. Nach Neutralisieren mit $\mathrm{KOH}$ wird erneut mit Äthanol/Kaliumacetat gefällt, zentrifugiert, in Wasser aufgenommen und gefriergetrocknet (Rohmucopolysaccharid-Peptide $\left.E^{\prime}\right)$. Die vereinigten überstehenden klaren Lösungen der 3 Äthanol/Kaliumaceta-Fällungen werden eingeengt, dialysiert und gefriergetrocknet (Fraktion C) **. 200-280 mg der Rohmucopolysaccharid-Fraktion $\mathrm{E}^{\prime}$ werden nach SCHILlER und Mitarb. 1. c. ${ }^{4}$ an Dowex 1-X2 200-400 mesh $\mathrm{Cl}^{\prime}$-Form (Säule $2,2 \times 120 \mathrm{~cm}$ ) chromatographiert. Hierzu wird $\mathrm{E}^{\prime}$ in ca. $10 \mathrm{ml}$ Wasser gelöst und mit etwa $50 \mathrm{ml}$ Wasser auf die Säule gespült. Anschließend wird mit $350-500 \mathrm{ml}$ Wasser und NaCl-Lösungen steigender Konzentration eluiert. Folgende Mengen werden im Durchschnitt benötigt:

$\begin{array}{rc}{[\mathrm{ml}]} & {[-n . \mathrm{NaCl}]} \\ 800-1100 & 0,5 \\ 900-1150 & 1,0 \\ 800-900 & 1,25 \\ 1300-1600 & 1,5 \\ 600-800 & 2,0 \\ 600-800 & 3,0\end{array}$

* Papain-Suspension von der Worthington Biochemical Corporation.

* Fraktion A, B und C sind uronsäurefrei. 
Die Fraktionen (ca. $15 \mathrm{ml}$ pro $30 \mathrm{~min}$ ) werden auf ihren Uronsäuregehalt getestet. Die einzelnen Fraktionen werden zusammengefaßt, gegen dest. Wasser chloridfrei dialysiert, gefriergetrocknet und ausgewogen. Die Aussagen über die Mucopolysaccharid-Peptide werden an Hand des Aminozuckerspektrums (Aminosäure-Analysator) erhalten.

Die vorliegenden Untersuchungen wurden in Zusammenarbeit mit Herrn Prof. Dr. Hauss und seinen Mit- arbeitern, Med. Klinik der Univ. Münster, durchgeführt, denen wir für die Bereitstellung des Aortenmaterials und für eingehende Diskussionen danken. Herrn Prof. Dr. Micheel sei für die Erlaubnis zur Benutzung des Aminosäuren-Analysators (Leihgabe des Landesamtes für Forschung des Landes NordrheinWestfalen) gedankt. - Dem Landesamt für Forschung des Landes Nordrhein-Westfalen und dem Verband der Chemischen Industrie „Fonds der Chemie" sind wir für die materielle Unterstützung zu Dank verpflichtet.

\title{
Zur Metachromasie von Toluidinblau in Gegenwart von Nucleinsäuren
}

\section{K. TEMPEL}

\author{
Institut für Pharmakologie, Toxikologie und Pharmazie der Tierärztlichen Fakultät \\ der Universität München (Vorstand: Prof. Dr. Dr. h. c. K. ZIPF) \\ (Z. Naturforschg. 24 b, 1279-1283 [1969] ; eingegangen am 23. April 1969)
}

1. Nach Zusatz von Nucleinsäuren zu wäßrigen Toluidinblaulösungen treten neue Farbqualitäten auf. Ihre Absorptionsmaxima lagen bei 655,585 und $555 \mathrm{~nm}$.

2. Art und Umfang dieser Metachromasie sind abhängig vom Reaktionsmilieu, vom Farbstoff-Polyanionen-Konzentrationsverhältnis sowie von Molekülgröße und Nativität der einzelnen Nucleinsäurepräparate.

3. In wäßrigen Proteinlösungen gelöste hochmolekulare DNS-Präparate verhalten sich gegenüber DNS-Lösungen ohne Proteinzusatz wie Präparate höherer Nativität.

4. Änderungen der metachromotropen Eigenschaften von DNS-Protein-Lösungen sind qualitativ und quantitativ als empfindlicher Nachweis für denaturierende Einflüsse (Hitze, ionisierende Strahlen, UV-Licht, Ultraschall, Tris- ( $\beta$-chloräthyl) -amin oder DNase I) verwertbar.

Das Wesen der Metachromasie besteht in der molekularen Wechselwirkung zwischen wäßrig gelösten kationischen Farbstoffen und anionischen Makromolekülen. Unter geeigneten Bedingungen kommt es zur genauen räumlichen Orientierung benachbarter Farbstoffmoleküle am Polyanion ${ }^{1-5}$. Daraus folgt, daß die metachromatische Reaktion qualitativ und quantitativ gewisse Rückschlüsse auf Art, Menge und sterische Anordnung der für die Reaktion verfügbaren anionischen Gruppen zuläßt ${ }^{6}$. Inwieweit dies auch für Nucleinsäuren gilt, wurde vorliegend am Beispiel der Metachromasie von Toluidinblau (TB) in Gegenwart verschiedener Desoxyribonucleinsäure (DNS) - und Ribonucleinsäure (RNS) -Präparate untersucht. Zur Denaturierung der DNS-Präparate dienten Hitze, Röntgenstrahlen, UVLicht, Ultraschall, Tris-( $\beta$-chloräthyl) -amin (Sinalost ${ }^{\circledR}$ ) und Desoxyribonuclease I (EC 3.1.4.5.) (DNase I).

1 M. Arnold, Histochemie 6, 1 [1966].

2 J.-P. Bidegaray et R. Viovy, J. Chim. physique 61, 1383 [1964].

3 J.-P. Bidegaray et R. Viovy, J. Chim. physique 61, 1391 [1964].

\section{Material und Methoden}

\section{M a terial}

1. Äthanol, 95-proz., mit Petroläther vergällt.

2. Albumin aus Hühnerei, Erg. B. 6, Riedel-DeHaen AG, Seelze.

3. Albumin aus Hühnerei, reinst, Serva, Nr. 11840.

4. Albumin aus Rinderserum, reinst, Serva, Nr. 11920.

5. Desoxyribonucleinsäure, Natriumsalz, aus Kalbsthymus, mind. 95\% DNS, MG $8900000, \mathrm{~N}$ ca. $14,5 \%$, P ca. $8,0 \%$, EGA-Chemie Keppler und Reif, Steinheim am Albuch (Präparat 1).

6. Desoxyribonucleinsäure aus Kalbsthymus, lyophilisiert, reinst, hochmolekular, rel. Viskosität ca. $3,0, t_{\mathrm{m}}$ mindestens $78{ }^{\circ} \mathrm{C}$, nach $\mathrm{Z}$ a m e $\mathrm{nh}$ of f, Serva Nr. 18560 (Präparat 2).

7. Desoxyribonucleinsäure aus Lachssperma, lyophilisiert, reinst, hochpolymer, praktisch protein- und salzfrei, $t_{\mathrm{m}}$ ca. $78{ }^{\circ} \mathrm{C}$, nach Emanuel und Ch ha i k of f, Serva Nr. 18570 (Präparat 3).

${ }^{4}$ R. Chayen and E. R. Roberts, Sci. J. Roy. Coll. Sci. 25, 50 [1955].

5 M. D. Schoenberg and R. D. Moore, Biochim. biophysica Acta [Amsterdam] 83, 42 [1964].

6 A. Krygier and K. Kasprzyk, Acta Med. Polona 2, 123 [1961]. 